\title{
TOMAR CAFÉ: REPRESENTACIONES SOCIALES Y PROCESOS DE SOCIALIZACIÓN SECUNDARIA EN CONTEXTOS LABORALES EN HABITANTES DE BOGOTÁ
}

\author{
Silvana Calle Vargas ${ }^{1}$ y Claudia Mercedes Padrón Mercado² \\ 1y 2 Fundación Universitaria Konrad Lorenz. Colombia. 1sicava_9@hotmail.com \\ 2claudiam.padronm@konradlorenz.edu.co
}

\begin{abstract}
Resumen. El objetivo del presente estudio es identificar las Representaciones Sociales y Procesos de Socialización Secundaria asociados a la toma de café en el contexto laboral en adultos entre los 35 a 50 años de la ciudad de Bogotá, teniendo en cuenta el significado simbólico de tomar café y las interacciones que esta actividad genera en el contexto colombiano. Se utilizó una metodología mixta secuencial que tuvo como primer momento una fase cualitativa en la cual se realizó una triangulación de fuentes y de técnicas para dar mayor credibilidad a la información y el método seleccionado fue el interaccionismo simbólico; el análisis se hizo mediante teoría fundamentada a través del software NVivo versión 11. En la fase cuantitativa, se recolectó la información de 201 consumidores de café a través de un formulario semiestructurado, para esta información, se realizó un análisis lexicométrico con SPSS y un análisis multidimensional con SPAD 9.0. Los resultados muestran los elementos del núcleo central resaltando los conceptos de activarse y energía como principales significados asociados al café; en el sistema periférico se identificaron características del café como el aroma y el sabor.
\end{abstract}

Palabras clave: Representaciones Sociales; Proceso de Socialización; Teoría Fundamentada; Análisis Multidimensional.

\section{DRINKING COFFEE: SOCIAL REPRESENTATIONS AND SECONDARY SOCIALIZATION PROCESSES IN LABOR CONTEXTS IN INHABITANTS OF BOGOTÁ}

Abstract: The objective of this study is to identify the social representations and secondary socialization processes associated with coffee drinking in the work context in adults between 35 and 50 years of age in the city of Bogotá, considering the symbolic meaning of coffee and coffee. interactions that this activity generates in the Colombian context. A sequential mixed methodology was used that had as a first stage a qualitative phase in which a triangulation of sources and techniques was carried out to give greater credibility to the information and the selected method was symbolic interactionism; the analysis was made through theory based on the Nvivo software version 11. In the quantitative phase, the information of 201 coffee consumers was collected through a semi-structured form, for this information, a lexicometric analysis was performed with SPSS and a multidimensional analysis with SPAD 9.0. The results show the elements of the central nucleus highlighting the concepts of activation and energy as main meanings associated with coffee; in the peripheral system, coffee characteristics such as aroma and flavor were identified.

Keywords: Social Representations; Socialization Process; Grounded Theory; Multidimensional Analysis. 


\section{INTRODUCCIÓN}

La llegada del café a Colombia tiene dos historias: La primera indica que el grano llega desde Centroamérica por Guatemala y Costa Rica; sin embargo, la segunda historia, sostiene a Venezuela como el canal por medio del cual entró el café al país, esta es la versión más creíble y probable dados los estudios realizados por José Charlaca en su libro "El café en la vida en Colombia" y la edición del mismo por parte de la Federación Nacional de Cafeteros (FNC) en el año 1987 (Durán y Barón, 2015). Según los historiadores que han escrito al respecto, el cultivo de café inicia en Santander con una tradición cultural asociada a las oraciones realizadas a "la virgen de los ojazos" quien, según sus seguidores, permitía la germinación de los frutos de café. No obstante, y secundando la anterior creencia, aparece un cura llamado Francisco Romero, quien, en el siglo XIX, solicita a los penitentes sembrar tanto café como pecados hubiesen cometido (Durán y Barón, 2015). Desde el año 1870 se comenzó a generar una producción comercial del producto, con un desarrollo, aunque lento, pero con una excelente reputación, debido a la estructuración y organización de pequeñas familias inscritas a la Federación Nacional de Cafeteros ${ }^{1}$, dedicadas al cultivo y recolección de café.

Este mercado ha despertado el interés por parte de diversos investigadores, puesto que el café en Colombia ha sido catalogado como la bebida más apetecida (Centro Nacional de Consultoría [CNC], 1993; 2009). Se ha estudiado la población penetrada por este mercado donde se descubre que, tomar café es una actividad que comienza en la adolescencia como parte de la motivación al iniciar la formación técnica o superior o al empezar la vida laboral, sin embargo, este consumo se destaca y aumenta marcadamente en relación con la edad, siendo los más grandes consumidores las personas mayores de 30 años y quienes reportan tomar al menos una taza de café al día (CNC, 2009; Sierra, 2013). En ese mismo sentido, es importante saber que, en relación con el consumo de café "la composición del grupo de consumidores en Colombia es similar a la pirámide socio demográfica, con una amplia base de consumidores en los estratos 2 y 3 cuyos motivos de consumo están asociados a la tradición y la costumbre" (Sierra, 2013, p.72).

\footnotetext{
${ }^{1}$ Federación Nacional de Cafeteros: Es una asociación que contribuye a promover el cultivo de café en Colombia y su exportación a mercados internacionales, regulando las políticas del sector.
} 
Es evidente como el consumo de café hace parte de las principales prácticas de los colombianos y funciona de manera identitaria y simbólica, siendo adoptada como una tradición o costumbre que se encuentra adscrita a interacciones en diferentes contextos y grupos sociales (FNC, 2010). Aunque el consumo de café en el país está ligado tanto a espacios laborales, como a espacios recreativos, "en el caso de Colombia, es un ritual ofrecer un café, al que se le llama 'tinto', a cualquier visitante a su hogar o lugar de trabajo, es a partir del café de donde surge la conversación" (FNC, 2010, p. 5), es por esto que los colombianos reconocen la importancia del producto para el país, aunque más que reconocerla, sienten que este producto los diferencia y les permite posicionamiento al generar una identidad cultural (Bejarano, 1980).

\subsection{Teoría de las Representaciones Sociales}

La teoría de las Representaciones Sociales aparece en Francia en los años 1960, a partir de formulaciones e investigaciones empíricas sobre los grupos sociales del mismo país a través de entrevistas, Moscovici (1988), como precursor de dicha teoría, la explica como una forma de entendimiento que permite construir comportamientos y comunicación entre personas para volver parte de la realidad física y social, la integración y los intercambios. Años después surge la idea sobre Representaciones Sociales como una forma de saber específico basado en el sentido común, en donde el pensamiento práctico está dirigido a la comunicación, el entendimiento y el control del medio social, físico y mental, un planteamiento construido por Denise Jodelet en el año 1986 (Banchs, 1986).

En su formulación sobre Representaciones Sociales, Jodelet afirma que estas, son imágenes que reúnen un grupo de conceptos que posibilitan interpretar y entender los sucesos de nuestra vida y que incluso, les dan sentido a aquellos eventos no esperados (Rubio, 2001).

Más adelante, Abric (2001), plantea que, "las representaciones sociales son conjuntos organizados y estructurados de creencias, opiniones, informaciones y actitudes en torno a un determinado objeto social y por tanto toda Representación Social posee una organización interna en la cual se presenta una jerarquización de los elementos que la componen y de las relaciones que se establecen entre ellos" (p. 8). El modelo de Abric, plantea un enfoque estructural que permite integrar en las investigaciones las características de las mismas y su organización alrededor de un núcleo central y un sistema periférico (Restrepo, 2013). 


\subsubsection{Núcleo central}

Es el sistema principal del modelo estructural de Abric, pues lo identifica y representa con características de los valores y creencias, es la parte esencial de las representaciones sociales, ya que a través de este, se determina el significado y la forma de organización de las mismas; en otras palabras, el núcleo central son aquellos elementos de las Representaciones Sociales que se mencionan o nombran en mayor medida, es decir, son las características que se refieren en repetidas ocasiones y que demuestran ser el componente más relevante del fenómeno estudiado.

El núcleo central está compuesto por tres factores determinantes: 1) la naturaleza del objeto representado, esto es, el origen o significado del elemento; 2) el vínculo que el sujeto o el conjunto de personas conserva con ese elemento y 3 ) el grupo de ideas (valores, normas) que caracteriza la unión entre el sujeto y el objeto, estos factores determinan la estructura y funcionamiento del sistema del núcleo central y lo mantienen (Abric, 2001).

\subsubsection{Sistema periférico}

Siendo la parte mediadora del enfoque estructural de Abric, defiende y funciona como protección del núcleo central, este sistema también tiene una organización jerárquica y actúa como elemento que aclara y justifica los significados más distantes al núcleo central, en otras palabras, el sistema periférico se diferencia porque la frecuencia de menciones sobre un significado es menor en relación con el núcleo central o son conceptos secundarios del mismo, aceptados y validados por las personas, aunque en menor medida reconocidos.

\subsection{Procesos de Socialización}

Berger y Luckmann (1968) plantearon una teoría que indica que los individuos se integran participando de la transformación de la sociedad, y es en el desarrollo biológico donde va introduciéndose a ella por medio de iniciadores o significantes. En ese mismo sentido, plantean que la socialización se puede clasificar en dos partes: Procesos de Socialización Primarios y Procesos de Socialización Secundarios. 


\subsubsection{Socialización Primaria}

La socialización primaria se refiere al proceso en el que el individuo se introduce en la sociedad por primera vez, en ella, los sujetos socializan casi que exclusivamente con su familia, sumergiéndose en un mundo objetivo como agente socializador. Esta etapa, sugiere ser la más importante para el individuo, en términos de aprendizaje y experimentación social (Berger y Luckmann, 1968).

\subsubsection{Socialización Secundaria}

Corresponde a la fase de la vida en la que se dan aspectos clave como la división y asignación del trabajo y la entrega social del conocimiento, por lo cual, esta se convierte en una fase necesaria en la vida de todo individuo, bajo esa perspectiva consideran que "la socialización secundaria es la internalización de 'submundos' institucionales o basados sobre instituciones. Su alcance y su carácter se determinan, pues, por la complejidad de la división del trabajo y la distribución social concomitante del conocimiento" (p. 53).

Basando este tipo de socialización en el aspecto laboral, Berger y Luckmann (1968), indican los argumentos y/o fundamentos para defender su posición teórica: "La socialización secundaria requiere la adquisición de vocabularios específicos de "roles", lo que significa, por lo pronto, la internalización de campos semánticos que estructuran interpretaciones y comportamientos de rutina dentro de un área institucional.

\section{METODOLOGÍA}

El estudio tuvo un diseño transversal mixto (Cualitativo/Cuantitativo), exploratorio, secuencial (DEXPLOS) con modalidad derivativa, que como lo plantea Hernández, Fernández y Baptista (2014), comienza con una fase cualitativa que buscó especificar características o propiedades del fenómeno social a estudiar, dado que permite tener un acercamiento al entorno natural y a la realidad; se partió de los resultados de esta fase para diseñar el instrumento cuantitativo. En el proyecto intervinieron dos (2) investigadores profesionales de las ciencias sociales y humanas.

La fase cualitativa se hizo a través del método de interaccionismo simbólico, haciendo énfasis en "los significados sociales que las personas asignan al mundo que les rodea" (Moreno, 2005, 
p. 75), y por medio de una triangulación de fuentes y de técnicas (Grupo focal, entrevistas en profundidad y observaciones no participantes).

La fase cuantitativa fue transversal, dado que la recolección de datos se realizó en un solo periodo de tiempo y permitió obtener una medición de manera independiente de las variables de estudio con precisión (Hernández, Fernández, y Baptista, 2014).

\subsection{Población}

En la fase cualitativa se invitaron a participar trabajadores o personas que tienen relación directa durante la preparación del café, o que tienen conocimiento de los rituales y formas en qué las personas se sirven e ingieren el producto, además, participaron expertos en el tema y consumidores frecuentes de café; en la fase cuantitativa se trabajó con habitantes de Bogotá, con edades entre los 35 a 50 años, laboralmente activos y consumidores frecuentes de café.

\subsection{Procedimiento fase cualitativa}

Para iniciar, se utilizó un muestreo por bola de nieve, dada la dificultad para contactar a los informantes clave, y se tuvo en cuenta el principio de saturación teórica que indica cuando "los datos se vuelven repetitivos o redundantes y el análisis confirma lo que se ha fundamentado". (Hernández, Fernández y Baptista, 2014, p. 435).

Se hizo una triangulación de técnicas, por medio de un (1) grupo focal con cinco personas que trabajan en el área de servicios generales de las empresas, cuatro (4) entrevistas en profundidad con expertos en temas de café y dos (2) observaciones no participantes en espacios de toma de café.

La información fue analizada por medio de teoría fundamentada, método por cual se determinaron categorías de análisis y ejes de indagación, que fueron extraídos a partir de una codificación abierta, axial y selectiva, a través del software de análisis cualitativo Nvivo versión 11. 


\subsection{Procedimiento fase cuantitativa}

Se usó un muestreo no probabilístico con un nivel de confianza del 95\% y un error muestral del $7 \%$, lo que arrojó una muestra total de 200 personas a encuestar.

Se aplicó una encuesta multimodal (cara a cara, telefónica o web), por medio de un cuestionario semiestructurado, conformado por 24 preguntas cerradas de selección múltiple y 10 preguntas abiertas, que en total tienen 122 ítems estructurados en 4 secciones: aspectos sociodemográficos, representaciones sociales, Procesos de Socialización y características de la toma de café.

Con los datos recopilados, se hizo un análisis lexicométrico por medio del software SPSS, con el fin de identificar la frecuencia de las variables, y también un análisis multidimensional y textual a través del software SPAD.

\section{RESULTADOS}

Para determinar el núcleo central y el sistema periférico de las Representaciones Sociales asociadas a la toma de café, se solicitó a los informantes que al frente de las tres palabras más mencionadas en la fase cualitativa: Café, charlar y activador de energía escribieran otras 6 palabras que asociaran con las antes mencionadas, y que, luego de ello, eligieran las dos más importantes para ellos y las definieran. Con esto, se realizó un análisis lexicométrico y un análisis de correspondencias múltiples para que en línea con el planteamiento de Abric (2001), se valoraran cada una de las asociaciones realizadas. Se presentan los resultados teniendo en cuenta las categorías orientadoras del estudio en donde se integran los resultados cualitativos (nodos de NVvivo) y los cuantitativos (frecuencias y segmentos de SPAD).

\subsection{Representaciones Sociales}

La primera dimensión representa los elementos del núcleo central de las Representaciones Sociales, está asociada con ACTIVAR, esta dimensión es descrita y adoptada por los participantes desde una perspectiva individual a través de la cual definen la toma de café, en la que desde la fase cualitativa y como se observa en la Figura 1 tiene mayor frecuencia y densidad, siendo el nodo con mayor número de menciones Tomar café como activador (33 
menciones en 5 recursos). De igual manera y como se observa en la Tabla 1, en la fase cuantitativa, se corrobora este elemento del núcleo central con una frecuencia relativa de $87,6 \%$ en el top two box (T2B) (Tomar café permite tener energía y activar el día a día).

La segunda dimensión asociada a la CHARLA, se identifica en la fase cualitativa con el nodo La charla como resultado de la toma de café (30 menciones en 7 recursos), este, describe la dimensión social o colectiva que tienen los individuos sobre la toma de café; y en la fase cuantitativa con una frecuencia relativa de $83,6 \%$ en el T2B, el ítem Compartir un café motiva a mantener una charla, apoya los resultados de la fase cualitativa. (Tabla 1)

Entre tanto, la tercera dimensión identificada es la COSTUMBRE, que representa para los informantes una dimensión cultural sobre la toma de café, descrita a través de los nodos La costumbre como motivo para tomar café (21 menciones en 5 recursos) y Tomar café como un hábito (9 menciones en 3 recursos), mientras que en el análisis estadístico con una frecuencia relativa de $86,6 \%$ y $85,1 \%$ en el T2B es identificada por medio de los ítems Tomar café es un hábito de mi vida y Tomar café permite seguir la cultura del país. (Tabla 1)

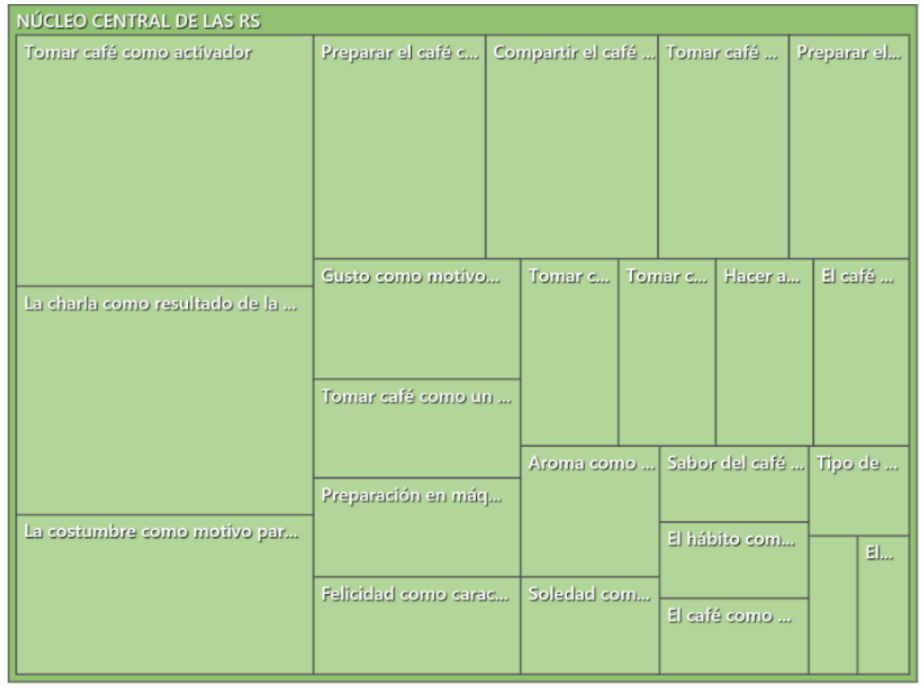

Figura 1. Núcleo central de las Representaciones Sociales asociadas a la toma de café. Fuente: Elaboración propia 
Tabla 1. Frecuencias variables - Núcleo central. Elaboración propia.

\begin{tabular}{llcc}
\hline Ítem & Tomar café permite tener energía y activar el día a día & $\begin{array}{c}\text { Mayor } \\
\text { frecuencia }\end{array}$ & $\begin{array}{c}\text { Menor } \\
\text { frecuencia }\end{array}$ \\
\hline 6 & Tomar café es un hábito de mi vida & 87,6 & 12,4 \\
11 & Tomar café permite seguir la cultura del país & 86,6 & 13,4 \\
8 & Compartir un café motiva mantener una charla & 85,1 & 14,9 \\
17 & 83,6 & 16,4 \\
\hline
\end{tabular}

En los elementos anteriores están los conceptos de Costumbre y Hábito que intentan describir la toma de café como parte de la cultura de los informantes, volviéndola un aspecto significativo del núcleo central de las Representaciones Sociales. También se observa, la Charla, que confirma el desarrollo de Procesos de Socialización mediante el núcleo central; y el concepto de Activar que describe el significado de la toma de café.

Lo anterior, permite entender el hecho que, para los participantes la toma de café en el contexto laboral, es en sí misma, un proceso de socialización secundaria.

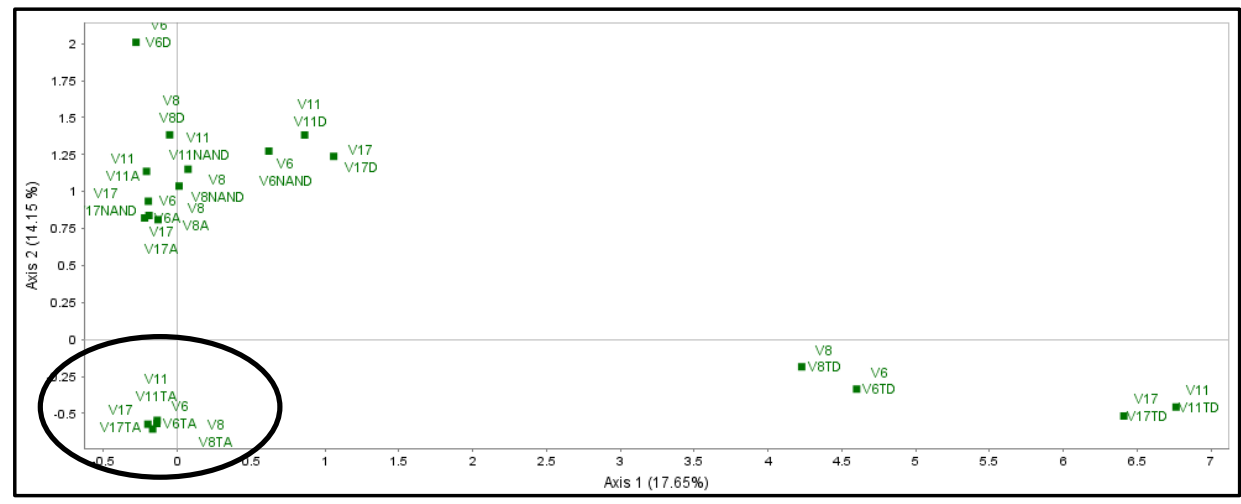

Figura 2. Análisis de correspondencias múltiples elementos del núcleo central. Fuente: Elaboración propia

Al hacer un análisis de correspondencias múltiples, como se puede observar en la figura 2, las variables de las Representaciones Sociales con mayor frecuencia están concentradas en los cuadrantes izquierdos y pertenecen a los siguientes ítems: (V6) Tomar café permite tener energía y actividad el día a día, (V8) tomar café permite seguir la cultura del país, (V11) tomar café es un hábito de mi vida, (V14) compartir un café permite conocer gente nueva, (V16) compartir un café es una forma de tener compañía y (V17) compartir un café motiva a mantener una charla; ítems que describen el núcleo central.

La información señalada en el círculo, muestra la concentración de las variables con opción de respuesta "Totalmente de acuerdo" que se encuentran cerca del promedio, esto significa que 
los ítems "Tomar café permite tener energía y activar el día a día”, "Tomar café permite seguir la cultura del país", "Tomar café es un hábito de mi vida" y "Compartir un café motiva a mantener una charla", son aquellos con mayor frecuencia en la primera opción de respuesta y representan 3 dimensiones: individual, social y cultural, anteriormente mencionadas.

La fase cualitativa permitió también identificar que a la toma de café se le atribuyen características como el sabor y el aroma para definir la importancia a la hora de tomarlo, aunque los participantes dejaron siempre clara la relevancia de la preparación del mismo para asignarle atributos de calidad. En la periferia de las Representaciones Sociales, se explican aspectos de las mismas asociadas con las características del café (olor, color, granos) y de la toma de café aludiendo a sus funciones, tanto individuales (trabajar, ánimo, felicidad, frío), como colectivas (chisme, interactuar, disfrutar), lo que apoya la descripción del núcleo central.

\subsection{Procesos de Socialización Secundaria}

Se observa en la figura 3 que, ejes de indagación como la Compañía y los Momentos ideales para la toma de café son descritos con variedad y mencionados con regular frecuencia por los participantes.

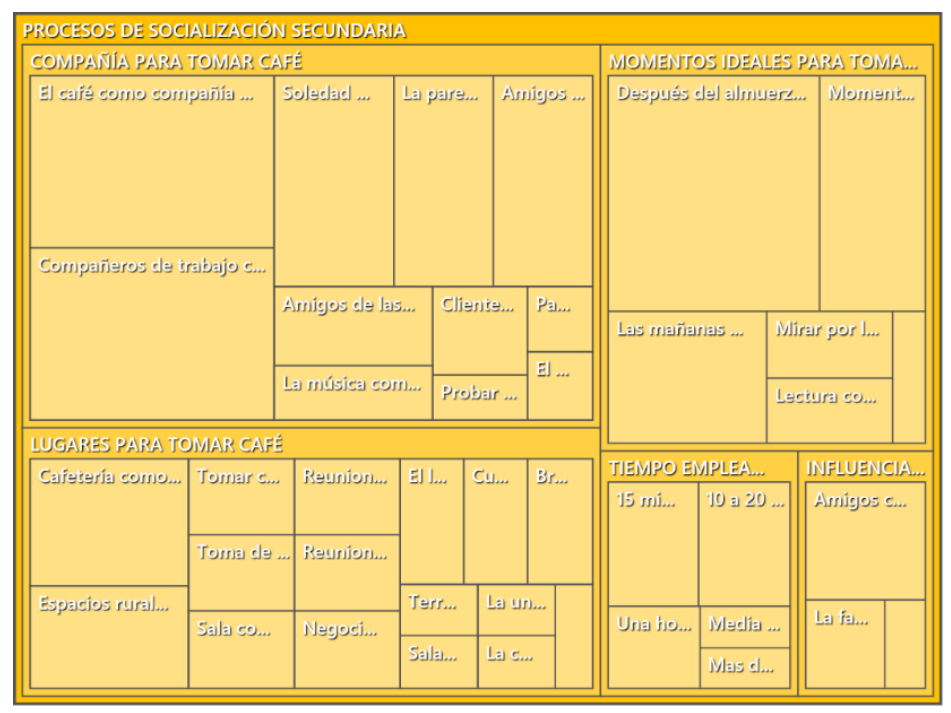

Figura 3. Procesos de Socialización Secundaria asociados a la toma de café. Fuente: Elaboración propia 
Analizando las concepciones de los informantes, definen a los compañeros de trabajo como la compañía para tomar café (10 menciones en 3 recursos) y con una frecuencia relativa de $74,1 \%$, aspecto que de cierta manera valida el hecho que, la toma de café en el contexto laboral genera Procesos de Socialización Secundaria; en sintonía con ello, la pareja como compañía ideal para tomar café y los amigos como compañía para tomar café ( 5 menciones en 3 recursos) y con una frecuencia relativa de 20,9\%, estos son dos códigos que apoyan la socialización secundaria, entendiendo a la pareja sentimental y a los amigos como relaciones sociales que se dan por fuera del núcleo familiar. Entre tanto, los amigos son descritos como los principales influenciadores de la toma de café (4 menciones en 2 recursos).

Por otro lado, los lugares para tomar café más mencionados por los informantes son diferentes al hogar, dejando claro que hay socialización secundaria en sus relaciones diarias; los espacios rurales con 4 menciones en 3 recursos, el sitio de trabajo y las cafeterías son los lugares que con mayor frecuencia se describen para el proceso de toma de café.

Aunado al núcleo central de las Representaciones Sociales, en donde la charla y el compartir un café hacen parte de las concepciones de los informantes sobre la toma de café en el contexto laboral, los Procesos de Socialización Secundaria se hacen evidentes por medio de lo que como ellos definen es un activador, una costumbre y un gusto.

Es importante hacer la claridad, de que, aunque se generan Procesos de Socialización Secundaria asociados a la toma de café, es por medio de los Procesos de Socialización Primaria que se adopta el hábito, la costumbre y dicho gusto por la toma de esta bebida. Los informantes describieron una serie de elementos importantes asociados a la toma de café por medio del proceso de socialización primaria, que según Berger y Luckmann (1968), son aquellas interacciones sociales generadas desde el nacimiento al interior de la familia. En su discurso, los participantes afirman, tomar la bebida en la mayoría de ocasiones porque desde la infancia en sus familias la bebían cuando iban a visitas o después de la alimentación, "desde pequeño veía como mis papás tomaban café todo el tiempo" - "mis papás me mandaban a hacer el café y servirlo después del almuerzo". 


\section{CONCLUSIONES}

Los resultados de la investigación permiten hacer un análisis bajo 3 dimensiones que explican las Representaciones Sociales y los Procesos de Socialización Secundaria asociados a la toma de café en el contexto laboral; una dimensión individual, donde se pueden enumerar y describir los beneficios funcionales de la toma de café, es aquí donde se encuentran los aspectos referidos por los informantes como "Tomar café como activador" o "Tomar café permite tener energía y activar el día a día", lo que describe las vivencias individuales a través de la toma de café. Se identifican una serie de elementos que atribuyen funciones a la bebida, en donde, tanto la representación como el proceso de socialización secundaria cumplen un importante papel dentro de dichas funciones, pues por un lado describen el significado que tiene para el informante y por el otro, lo que puede hacer con él en su lugar de trabajo y su vida cotidiana.

Una dimensión social, que permite entender la estrecha relación que existe entre la toma de café y los Procesos de Socialización, en donde esta actividad da la posibilidad de "mantener una charla", "conocer gente nueva" y "hacer amigos", como lo describen en su discurso los informantes; identificando en sí misma a la toma de café como un proceso de socialización tanto primaria como secundaria. En esta dimensión, sobresalen aspectos colectivos de los individuos, como las necesidades y deseos de interactuar con otras personas y la oportunidad que ven en la toma de café para hacerlo.

Y una dimensión cultural caracterizada por la descripción de la toma de café como parte de las costumbres familiares y los aprendizajes de los Procesos de Socialización Primaria, en donde nodos como "Tomar café como un hábito" y "La costumbre como motivo para tomar café", concluyeron la exposición del café como componente cultural de los informantes.

Si bien la investigación tenía como foco hablar de los Procesos de Socialización Secundaria, debido a que se estaba trabajando con población adulta, se identificó al preguntar por el proceso de la toma de café y sus preferencias en relación con este, que, los informantes lo asocian con la forma como aprendieron a tomarlo, dado que en su discurso, mencionan experiencias como la preparación del café en la infancia, el consumo de este acompañado de complementos como pan, arepa, torta, entre otros y la observación de reuniones familiares en las que se compartía un café para conversar. 
Lo anterior permitió identificar los Procesos de Socialización Primaria, como una categoría emergente dentro de la investigación, a través de la cual se narraron historias sobre el aprendizaje de la toma de café y el mismo como parte de los hábitos y prácticas culturales de la familia, todo esto, desde vivencias de la infancia.

Las tres dimensiones identificadas y descritas, son una manera de comprender como algo como una representación social instaura y mantiene una práctica de consumo como la toma de café:

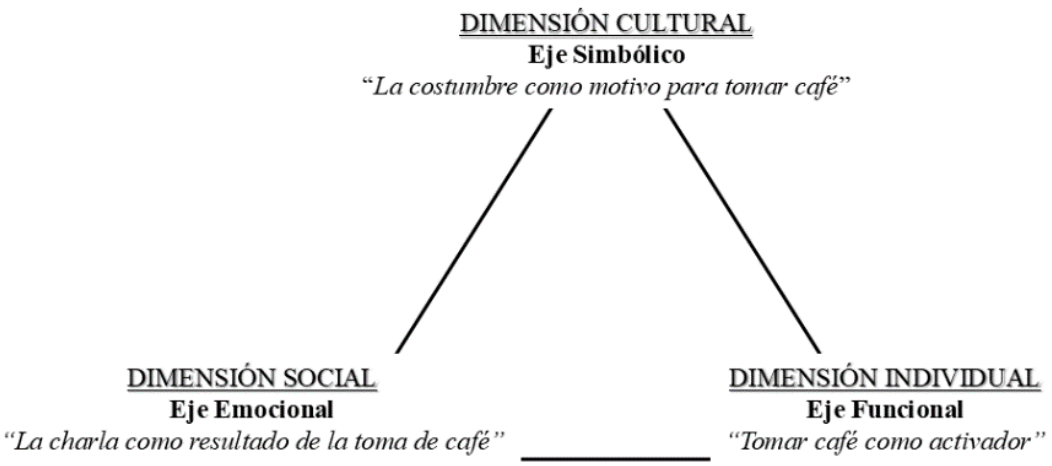

Figura 4. Dimensiones de las Representaciones Sociales y los Procesos de Socialización asociadas a la toma de café. Fuente: Elaboración propia

Las Representaciones Sociales y los Procesos de Socialización generan un tejido social que se retroalimenta en ambas vías, lo social mantiene los significados de la toma del café y las representaciones se mantienen a través de la socialización que se afianza en espacios laborales a través de la misma.

Teniendo en cuenta lo anterior, y tal como lo describe Perera (2003, p. 5), las Representaciones Sociales son "una forma específica de pensamiento social que tiene su origen en la vida cotidiana de las personas, al tiempo que otorga al pensamiento social una importante función en la estructuración de la realidad social"; así, y en línea con las 3 dimensiones halladas en el presente estudio, el eje simbólico y el eje emocional constituyen el pensamiento colectivo que origina la cotidianidad de los informantes, y el eje funcional estructura su realidad.

Entre tanto, Bordieu y Wacquant (2005, p. 31) plantearon "la objetividad del primer orden", en la cual describen la sociedad como una estructura objetiva que tiene características materiales 
organizadas según la representación que se hagan quienes viven en ella; lo que en el orden de lo individual, social y cultural puede explicar dicha objetividad de la sociedad.

Aunado a lo anterior y contemplando el planteamiento de Abric (2001, p. 45) los elementos centrales de las Representaciones Sociales tienen tres características que se asocian directamente con las tres dimensiones resultado del proyecto:

1) Un valor simbólico en el sentido que "un elemento central no puede ser cuestionado sin afectar la significación de la representación"; lo que, de cierta manera, se relaciona con la dimensión cultural y como tal con el eje simbólico que describe las costumbres, hábitos y Procesos de Socialización Primaria, que generan en el individuo unas representaciones iniciales desde la infancia y que por mucho permanecen en sus actividades cotidianas. (Codificación selectiva)

2) Un valor asociativo en la medida en que "un elemento central está directamente vinculado con la significación de la representación, está necesariamente asociado con un amplio número de constituyentes de la representación"; está relacionado con la dimensión social, por ende, con el eje emocional, en el cual el imaginario colectivo impide en muchas ocasiones separar los significados individuales de los comunes. (Codificación axial)

3) Un valor expresivo que se manifestaría a través de la frecuencia de aparición de un término, aunque complementando esta apreciación con información más cualitativa; este valor se asocia directamente con la dimensión individual, esto es, con el eje funcional, del cual se mencionó con mayor frecuencia tanto en la fase cualitativa como en la fase cuantitativa los beneficios de la toma de café, específicamente, el hecho de ser una bebida que permite activar o generar energía. (Codificación abierta)

Estudios como el presente permiten conocer la importancia e interés en realizar investigaciones sobre elementos culturales de un país con otros tipos de alcances, por ejemplo, comparar diferentes poblaciones en donde existan condiciones que puedan afectar la socialización a través del café, monitorear (a través de un estudio de corte longitudinal) en diferentes grupos etarios los significados y la manera como se interactúa por medio de la toma de café o indagar elementos que mantienen las prácticas de consumo en personas que toman café y aquellas que no lo consumen. 
Anexos: En el siguiente link podrán descargar los instrumentos de recolección de información cualitativa y cuantitativa:

https://drive.google.com/drive/folders/1UqjNSJ7kfjoeR4ncsHqr-B9GUXBBPIcS?usp=sharing

\section{REFERENCIAS}

Abric, J.-C. (2001). Prácticas sociales y representaciones. Francia: Ediciones Coyoacán, S.A de C.V.

Banchs, M. (1986). Concepto de representaciones sociales: Análisis comparativo. Revista Costarricense de psicología, 27-40.

Bejarano, J. A. (1980). Estudios sobre la historia del café en Colombia. Cuadernos de Economía, 1 (2), 115-140.

Berger, P y Luckmann, T. (1968). La construcción social de la realidad. Buenos Aires: Amorrortu.

Bordieu, P., y Wacquant, L. (2005). Una invitación a la sociología reflexiva. Buenos Aires: Siglo XXI Editores Argentina S.A.

Centro Nacional de Consultoría. (1993). Estudio sobre el consumo de café en Colombia. FEDERACAFE: Estudios Especiales, 71-83.

Centro Nacional De Consultoría. (2009). Evaluación de la percepción del café en la consulta médica. Bogotá.

Durán, M. J., y Barón, A. (2015). El impúdico brebaje: Los cafés de Bogotá. Bogotá: Instituto Distrital de Patrimonio Cultural. Bogotá Humana.

Federación Nacional de Cafeteros. (2010). Café de Colombia. Recuperado el 24 de septiembre de 2017, de Federación Nacional de Cafeteros:

http://www.cafedecolombia.com/particulares/es/sobre_el_cafe/mucho_mas_que_una_bebida/ritos_y_costum bres/

Hernández, A., Fernández, C., Baptista, F. (2014). Metodología de la Investigación. Ciudad de México: McGraw Hill Interamericana Editores, S.A de C.V.

Monsalve, D. (1927). Colombia cafetera. Madrid: Barcelona, Artes Gráficas, S.A., Sucesores de Henrich y Cia.

Moreno, M. C. (2005). La investigación cualitativa en marketing: el camino hacia la percepción social del mercado. Revista colombiana de marketing, 4 (6), 68-85.

Moscovici, S. (1988). Notes towards a description of social representations. European Journal of Social Psychology, 211-250.

Perera, M. (2003). A propósito de las representaciones sociales: apuntes teóricos, trayectoria y actualidad. Consejo Latinoamericanos de Ciencias Sociales CLACSO, 1-35.

Restrepo, D. A. (2013). La Teoría Fundamentada como metodología para la integración del análisis procesual y estructural en la investigación de las Representaciones Sociales. Revista CES Psicología, 122-133.

Rubio, F. D. (2001). Teoría de las Representaciones Sociales. Apuntes. Revista Crítica de Ciencias Sociales y Jurídicas. Volumen (3), p. 1-16.

Sierra, A. M. (2013). Actitudes y hábitos de consumo de café en Colombia: Tradición y Bienestar. Alimentos Hoy: Revista de la asociación colombiana de ciencia y tecnología de alimentos, 71-75. 